\section{Conturic}

Correspondence to Dr Peter Clark, Leukocyte Biology Section, National Heart and Lung Institute, Imperial College London, Sir Alexander Fleming Building, South Kensington Campus, London SW7 2AZ, UK; p.clark@imperial.ac.uk

Received 30 September 2013 Revised 27 October 2013 Accepted 1 November 2013 Published Online First 13 November 2013 \title{
Protease-mediated ectodomain shedding
}

\author{
Peter Clark
}

ABSTRACT

Ectodomain shedding is the proteolytic cleavage of cell surface proteins resulting in the loss of the extracellular domains. This mechanism is important in a variety of normal and pathological processes, including growth factor signalling, cell adhesion, inflammation and cell survival. Elevated protease activity in the lungs is a key pathological mechanism in emphysema which could enhance ectodomain shedding in lung cells. Here, the major steps and consequences of ectodomain shedding are reviewed.

Ectodomain shedding is a key event in many developmental and homeostatic processes, and also in a number of important pathologies. ${ }^{1}{ }^{2}$ Cell membrane proteins undergo proteolytic cleavage at the cell surface, resulting in the release of a significant portion of their extracellular domain, the ectodomain (figure 1). This cleavage leads to the shedding of the free ectodomain while the cell retains the membrane-associated remnant peptide (eg, the transmembrane stub of the original protein), which itself can undergo subsequent proteolytic cleavage. Proteins that are subject to shedding include cell adhesion molecules (cell-cell and cell-matrix), growth factor and cytokine transmembrane precursors and receptors, cell-surface enzymes, and others. Indeed, it has been suggested that all membrane proteins might be subject to shedding to some extent. However, the best understood examples are proteins for which ectodomain shedding is part of their physiological function or turnover (eg, Notch signalling in many developmental and cell differentiation processes, including T-cell maturation; and also signalling by epidermal growth factor (EGF) family molecules), or is part of a well characterised pathology (eg, amyloid precursor protein (APP) in Alzheimer's disease). In this issue, Mimae $e t \mathrm{al}^{3}$ report on an ectodomain shedding event implicated in stimulating apoptotic cell death in emphysema. Here, the basic biology of ectodomain shedding is reviewed.

\section{SHEDDASES}

Any protease that cleaves a protein to release the extracellular domain can be said to have sheddase activity. ${ }^{2}{ }^{4}$ Sheddases are also known as secretases, since they lead to the release of proteins and peptides from the cell surface. However, sheddase activity may not always be physiologically or pathologically relevant, and could be the result of bystander activity in unusual circumstances, for example, a number of the proteolytic cleavage events seen in emphysema may not be biologically significant or contribute to the pathology.
The first 'true' sheddase characterised was the transmembrane enzyme responsible for cleaving and releasing soluble, active tumour necrosis factor $\alpha$ $(\mathrm{TNF} \alpha)$ from its inactive, transmembrane precursor. This TNF $\alpha$-converting enzyme is also known as ADAM17. The ADAMs (a disintegrin and metalloproteinase) are a family of transmembrane proteases that are considered to be a major class of sheddases. Another family of metalloproteinases, the matrix metalloproteinases (MMPs), also play essential roles in ectodomain shedding. Some members of this family, the membrane type MMPs (MT-MMPs), directly associate with the plasma membrane as transmembrane proteins or via lipid anchors. Secreted MMPs may also reside at the cell surface through binding to other cell surface proteins.

The amino acid sequence at the cleavage site of a sheddase-susceptible protein appears not to determine the site's position. It is believed that distances from the plasma membrane and the secondary structure are the important determinants. There is also a degree of promiscuity in ectodomain shedding in that an individual sheddase may act on many proteins, and an individual membrane protein may be cleaved by a number of sheddases. For example, ADAM17 can cleave TNF $\alpha$, TNF $\alpha$ receptors, APP, L-selectin and others, and the epithelial intercellular junction adhesion molecule, E-cadherin, can be cleaved by ADAM10, ADAM15, MMP7, MMP9 and others. In addition to the endogenous sheddases, pathogen-derived protease activity can lead to ectodomain shedding, which may play an important role in disease progression.

\section{CONSEQUENCES OF ECTODOMAIN SHEDDING}

Clearly, the function of the original protein will determine the activity of shed ectodomains (figure 1). ${ }^{1}{ }^{2} 5$ The ectodomain may be inert (eg, the cleaved Notch ectodomain is internalised and degraded) or have significant biological activity. Downregulation of a functional receptor or adhesion molecule at the cell surface is an effect in itself. Ectodomain shedding of active growth factors from cell surface precursors will result in increased signalling, while shed growth factor receptor ectodomains will reduce signalling by acting as decoys that bind and neutralise extracellular growth factors. Similarly, soluble cell adhesion molecule ectodomains may interfere with cell-cell adhesion by binding to intact cell adhesion molecules at the cell surface.

\section{FATE OF THE REMNANT PEPTIDE}

The short transmembrane remnant that remains after ectodomain shedding (the C-terminal 


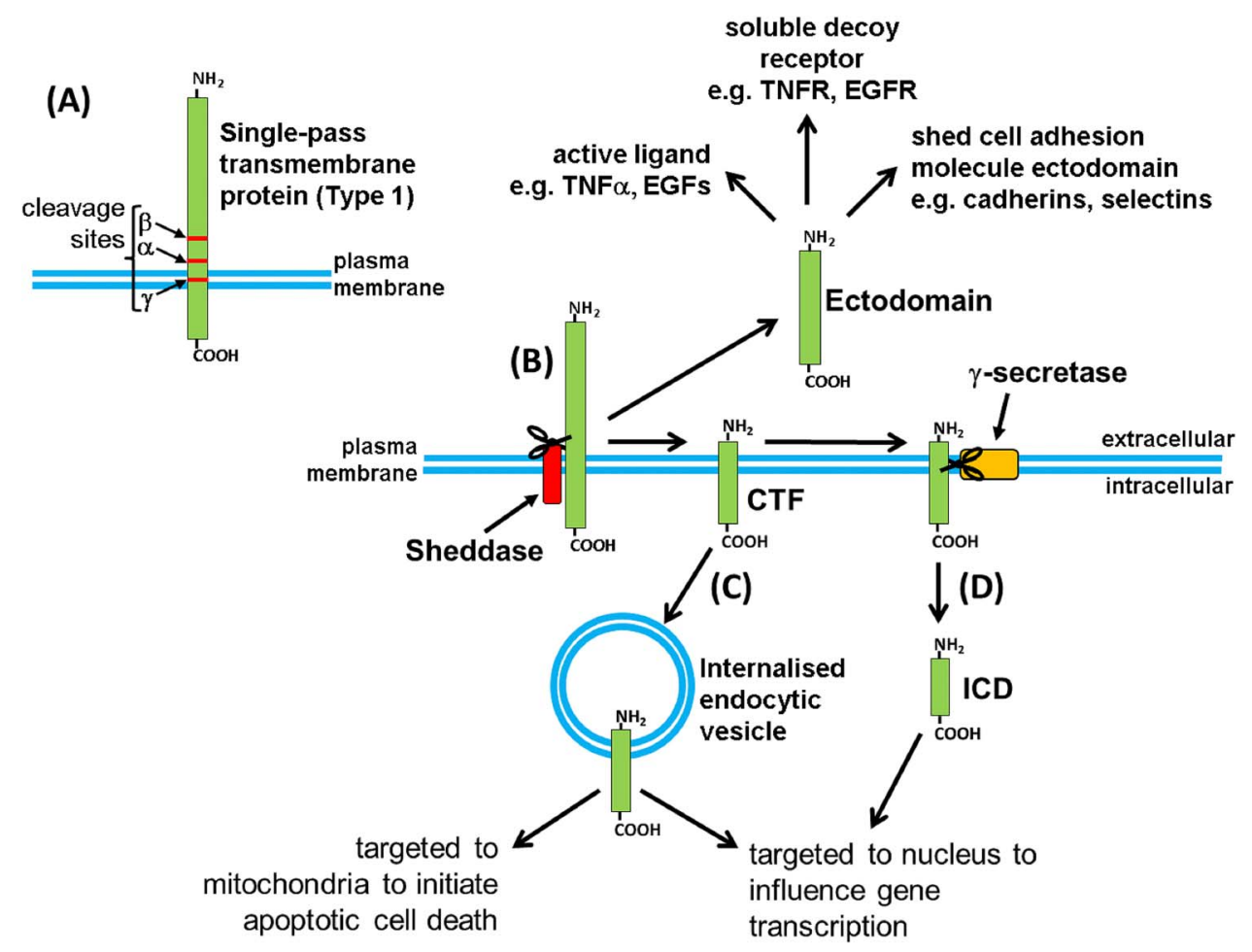

Figure 1 (A) The position of ectodomain shedding cleavage sites, based on the amyloid precursor protein (APP) which is a type 1 transmembrane protein, that is, single span with a cytoplasmic C-terminal. In this issue, Mimae et al ${ }^{3}$ use this cleavage site classification for cell adhesion molecule 1 (CADM1). Cleavage at the $\alpha$ site will produce a larger ectodomain fragment and smaller C-terminal fragment (CTF) than $\beta$ cleavage. The $\gamma$ cleavage site is where intramembrane proteolysis occurs to give rise to an intracellular domain (ICD) peptide. The nomenclature of cleavage sites can vary for other proteins, for example, $\alpha$ and $\gamma$ equivalent sites in Notch protein are S2 and S3, respectively. (B) Sheddase cleavage at a juxtamembrane site will produce a shed ectodomain and CTF. The function, if any, of the ectodomain depends on the protein of origin. (C) The intact CTF can be endocytosed and targeted to various intracellular compartments. (D) The CTFs of type 1 proteins are commonly subject to $\gamma$-secretase cleavage to release an ICD into the cytoplasm. Intramembranous proteolysis of type 2 proteins is carried out by signal peptide peptidase-like (SSPL) enzymes. ICDs can associate with other cytoplasmic proteins to form complexes that enter the nucleus and act as transcription factors controlling gene transcription and thereby cell function. EGF, epidermal growth factor; TNF $\alpha$, tumour necrosis factor $\alpha$.

fragment (CTF) for type 1 proteins) may be internalised by endocytosis (figure 1C) or may undergo further cleavage (figure 1D). ${ }^{15}$ After ectodomain cleavage, some remnant peptides become susceptible to regulated intramembranous proteolysis, mediated by intramembrane-cleaving proteases, for example, $\gamma$-secretase, to produce an intracellular domain (ICD) that is released into the cytoplasm (figure 1D). The internalised CTF and the ICD can be targeted to intracellular compartments where they influence cell function. For example, the basis of Notch signalling is that the ICD enters the nucleus and affects gene transcription. In this issue, Mimae et $a l^{3}$ show that the CTF of cell adhesion molecule 1 (CADM1) is targeted to, and disrupts, mitochondria, inducing apoptotic cell death.

\section{REGULATION OF ECTODOMAIN SHEDDING}

Although some proteins might be continuously shed at low levels, ectodomain shedding is typically subject to regulation. ${ }^{6}$ This regulation may be at the level of expression of sheddases and their targets, and also their targeting to distinct plasma membrane domains. ADAM and MMP activities can be modulated by tissue inhibitors of MMPs (TIMPs). Sheddases themselves can be activated or degraded by other proteases. However, cell signalling events are known to alter ectodomain shedding. This may be the result of sheddase activation or a change to the target protein making it more susceptible to cleavage. The details of these control mechanisms are not clear, though $\mathrm{Ca}^{2+} /$ calmodulin activity, protein kinase $\mathrm{C}$ (PKC) activation and tyrosine phosphorylation have all been implicated in inducing ectodomain cleavage, possibly by promoting specific sheddase/target interactions. In Notch signalling, it is believed that the binding to its ligand on an adjacent cell leads to exposure of a previously cryptic cleavage site. It is not clear if this type of control is used by other proteins. Some pathogens stimulate ectodomain shedding indirectly (ie, not by direct proteolysis). For example, the Pseudomonas aeruginosa virulence factor, LasA, is a protease that does not directly stimulate ectodomain shedding, but stimulates host sheddase activity that results in the cleavage of a host protein, syndecan-1. This heparan-sulfated syndecan-1 ecotodomain can bind and neutralise chemokines involved in lymphocyte cell recruitment to the lung, thereby blunting the immune response.

\section{ECTODOMAIN SHEDDING IN PATHOPHYSIOLOGY}

Given that it is central to a number of fundamental signalling and adhesion processes, it is not surprising that ectodomain shedding is important in many pathologies. The need to dissect the molecular and cellular mechanisms of the proteolytic processing of APP that produce amyloid in Alzheimer's disease is a major driving force in understanding ectodomain shedding. Similarly, its role in immunity and inflammation implicates shedding in many pathological states. Normal pulmonary and cardiovascular development, cancer and other pathologies are strongly dependant on growth factor signalling, which is modulated by ectodomain shedding. Proteolytic cleavage of 
cell-surface adhesion molecules is increasingly linked with tumour progression and metastasis, and plasma levels of ectodomains can act as prognostic markers.

There are numerous examples of the importance of ectodomain shedding in the lung. In mouse models, knocking out the sheddase, ADAM17, has strikingly similar effects on lung development to knocking out TNF $\alpha$, EGF or their receptors. The impaired airway branching and pulmonary epithelial defects observed emphasise the importance of ectodomain shedding in the normal control of growth factor activity. In chronic lung infection, lipoteichoic acid, released from Staphylococcus aureus, induces the activation of ADAM10, which cleaves and sheds EGF, which in turn stimulates mucus production, contributing to airway obstruction. Genome-wide association studies implicate ADAM33 in asthma and airway hyper reactivity. The mechanisms by which it acts are not clear, but ADAM33 can cleave and shed CD23, the FceRII low-affinity IgE receptor, and is therefore likely to influence responses to allergens. In addition, transforming growth factor $\beta$, a key factor in tissue remodelling that is upregulated in asthma, stimulates ectodomain shedding of ADAM33, which may deregulate its proteolytic activity and contribute to the remodelling process seen in asthma. MMP7 (matrilysin) is upregulated in lung fibrosis when it increases ectodomain shedding of E-cadherin from epithelia, leading to loss of epithelial coherence, and increased migration by epithelial cells, part of the epithelial repair response found in fibrosis. Emphysema is a disease for which protease activity is elevated in the lung, and it is likely that ectodomain shedding could play a significant part in its pathology. Mimae et $a l^{3}$ show that the ectodomain shedding of CADM1 in pulmonary epithelial cells results in apoptosis, and the elevated CADM1 shedding that they observed in emphysematous patient samples suggests that shedding might be a central mechanism in the loss of alveolar septa.

\section{CONCLUSION}

Ectodomain shedding is under considerable scrutiny in relation to developing therapies for neurodegenerative diseases, cancer and inflammation. The study by Mimae et $a l^{3}$ in this issue is one of what is likely to prove to be a long list of lung pathologies involving ectodomain shedding. Investigating the membrane proteins involved, their sheddases, and the mechanisms regulating cleavage and shedding will provide important targets for therapeutic intervention.

Competing interests None.

Provenance and peer review Commissioned; internally peer reviewed.

\section{REFERENCES}

1 Hayashida $\mathrm{K}$, Bartlett $\mathrm{AH}$, Chen $\mathrm{Y}$, et al. Molecular and cellular mechanisms of ectodomain shedding. Anat Rec (Hoboken) 2010;293:925-37.

2 Weber S, Saftig P. Ectodomain shedding and ADAMs in development. Development 2012;139:3693-709.

3 Mimae T, Hagiyama M, Inoue T, et al. Increased ectodomain shedding of lung epithelial cell adhesion molecule 1 as a cause of increased alveolar cell apoptosis in emphysema. Thorax 2014;69:223-31.

4 Chow FL, Fernandez-Patron C. Many membrane proteins undergo ectodomain shedding by proteolytic cleavage. Does one sheddase do the job on all of these proteins? IUBMB Life 2007;59:44-7.

5 Higashiyama S, Nanba D, Nakayama $H$, et al. Ectodomain shedding and remnant peptide signalling of EGFRs and their ligands. J Biochem 2011;150:15-22.

6 Hartmann M, Herrlich A, Herrlich P. Who decides when to cleave an ectodomain? Trends Biochem Sci 2013;38:111-20. 